\title{
Influência de tratamentos termo-mecânicos na estrutura e microestrutura da liga Ti-10\%pNi obtida por fusão a arco
}

\author{
Daniela Cascadan ${ }^{1}$, Carlos Roberto Grandini ${ }^{2}$
}

\author{
UNESP - Univ. Estadual Paulista, Laboratório de Anelasticidade e Biomateriais, 17033-360, Bauru, SP \\ ${ }^{1}$ e-mail: dcascadan@fc.unesp.br \\ 2 e-mail: betog@fc.unesp.br
}

\begin{abstract}
RESUMO
Ligas de titânio representam uma importante classe de biomateriais. Além da questão da biocompatibilidade, é muito importante considerar as propriedades mecânicas de um biomaterial, que são diretamente relacionadas com a microestrutura e quantidade de elementos intersticiais presentes. No presente trabalho, a liga Ti-10\%pNi foi produzida a partir da fusão dos metais titânio e níquel comercialmente puros, num forno de arco sob uma atmosfera de argônio. Em seguida, foi realizado um tratamento térmico e a amostra foi laminada. Para comprovar a composição da liga, foi realizada análise química quantitativa. A estrutura e microestrutura da liga produzida foram analisadas por difração de raios-x, microscopia óptica e microscopia eletrônica de varredura. Os resultados mostram que a liga apresenta predominantemente a fase alfa do titânio (estrutura hexagonal compacta), com precipitados lamelares proeutetóide numa matriz eutetóide composta de fase alfa e do intermetálico Ti2Ni.
\end{abstract}

Palavras-chave: Liga de titânio, microestrutura, propriedades mecânicas, processamento.

\begin{abstract}
Titanium alloys are an important class of biomaterials. Apart from the issue of biocompatibility, is very important to consider the mechanical properties of a biomaterial, which are directly related to the microstructure and interstitial elements quantity present. In the present paper, Ti-10wt\%Ni was produced from the melting of commercially pure titanium and nickel in arc furnace under argon atmosphere. Then a heat treatment was carried out and the sample was hot-rolled. To prove the alloy composition, quantitative chemical analysis was performed. The structure and microstructure of the produced alloy were analyzed by $\mathrm{x}$-ray diffraction, optical and scanning electron microscopy. The results show that the alloy presents predominantly titanium alpha phase (hexagonal compact structure), with proeutectoid lamellar precipitates in eutectoid matrix of alpha phase and intermetallic Ti2Ni.
\end{abstract}

Keywords: Titanium alloy, microstructure, mechanical properties, processing.

\section{INTRODUÇÃO}

A pesquisa sobre novos biomateriais é de fundamental importância, pois com o aumento e envelhecimento da população mundial, a demanda tem aumentado substancialmente [1]. Neste sentido, o titânio e suas ligas têm sido muito utilizados como materiais biomédicos, em função da alta resistência à corrosão, ótima relação resistência mecânica/densidade e excelente biocompatibilidade [2, $\underline{3}]$ Ligas de titânio usadas tradicionalmente em próteses, como Ti-6Al-4V, apresentam falhas como liberação de íons tóxicos e propriedades mecânicas não muito satisfatórias como módulo elástico alto (cerca de $112 \mathrm{GPa}$ ) em comparação com o do osso (cerca de $30 \mathrm{GPa})$ devido à sua microestrutura do tipo $\alpha+\beta[\underline{4}, \underline{5}]$. No caso de implantes ortopóedicos, ligas com microestrutura $\beta$ são mais apropriadas devido ao menor módulo elástico, cerca de 60-80 GPa [2] .

No entanto, ligas de titânio com fase $\alpha$, apesar de apresentarem maior módulo elástico, apresentam melhor oxidação, resistência à corrosão e soldabilidade, comparado com as ligas $\beta$ []ㅡ. Assim, uma série de

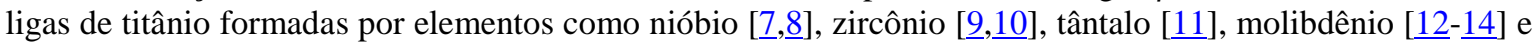
níquel [15] tem sido investigadas nos últimos anos, visando substituir a liga Ti-6Al-4V. Estas ligas apresentam propriedades que variam de acordo com o tipo de elemento de liga e sua concentração, além de sofrerem alterações significativas devido à presença de elementos intersticiais como nitrogênio e oxigênio, que também provocam distorções na rede cristalina $[\underline{10}, \underline{12}]$. 
Como as propriedades mecânicas das ligas de titânio são fortemente dependentes da microestrutura, para se conseguir uma resistência mecânica adequada a seu propósito, as microestruturas desejadas somente são conseguidas por meio de etapas controladas e adequadas de processamento mecânico e tratamentos térmicos. Tratamentos térmicos com a liga permanecendo várias horas em temperaturas elevadas seguida de baixa taxa de resfriameto permitem o crescimento das microestruturas devido ao processo de difusão atômica em larga escala [] ]. Tratamentos de conformação mecânica são importantes para tornar possíveis ensaios que requeram uma amostra simétrica, porém, estes alteram a microestrutura ao deformar o material originado tensões internas []].

Algumas ligas do sistema Ti-Ni podem ser consideradas como biomaterial com alto potencial para serem utilizadas em aplicações ortopédicas tais como implantes de joelho e quadril. Este material satisfaz em maior grau os requisitos e adicionalmente apresenta características superiores que permitem o longo tempo de uso do material no corpo, incluindo efeito memória de forma e superelasicidade, além de biocompatibilidade por curtos períodos de tempo e boas propriedades de corrosão, com propriedades similares (baixo módulo de Young, resistência à fadiga e ductibilidade) e até mesmo superiores às do aço inoxidável, ligas Co-Cr, Ti-Al-V e Ti-cp [4]. Apesar do níquel apresentar diversas reações adversas no organismo tais como efeitos carcinogênicos, genotóxico, mutagênico, citotóxico, alérgicos dentre outros [16], contudo, testes de corrosão simulando ambientes naturais no organismo mostraram que ligas Ti-Ni $(54,5-$ $57 \%$ pNi) apresentaram liberação de níquel em níveis seguros, muito inferior à ingestão humana diária ${ }^{17 ; 18}$, além dos testes de biocompatibilidade in vitro e in vivo com resultados positivos $[\underline{19}, \underline{20}]$.

Em ligas Ti-Ni com baixa concentração de níquel, devido à reação eutetóide $\beta \rightarrow \alpha+\mathrm{Ti}_{2} \mathrm{Ni}$ a $6 \% \mathrm{pNi}$, geralmente apresentam precipitados proeutetóide numa matriz de microestrutura eutetóide [21,22]. Com a adição de Ni ao titânio, a resistência mecânica e à corrosão é aumentada, tornando estas ligas próprias para uso de dispositivos de fixação em materiais para implante [23].

Este trabalho mostra a preparação, caracterização estrutural e microestrutural e o efeito de tratamentos térmicos e mecânicos na estrutura e microestrutura da liga Ti-10\%pNi, com vistas à sua utilização biomédica.

\section{MATERIAIS E MÉTODOS}

A liga Ti-10\%pNi foi produzida a partir da fusão do titânio e níquel comercialmente puros, num forno à arco, sob uma atmosfera inerte de argônio, obtendo-se um lingote de aproximadamente $120 \mathrm{~g}$.

A análise química foi realizada no Centro de Caracterização e Desenvolvimento de Materiais (CCDM) da UFSCar. Os elementos de interesse foram determinados em espectrômetro de emissão óptica com plasma induzido, modelo VISTA, da marca Varian (AQ-206 rev.012), a partir da solubilização em meio ácido. Para verificar a homogeneidade das amostras produzidas, foi efetuado o mapeamento dos elementos constituintes por espectroscopia de energia dispersiva (EDS), utilizando um equipamento Inca Energy 250, da Oxford. A análise dos teores dos elementos intersticiais oxigênio e nitrogênio, foi realizada no Laboratório de Metalurgia Física e Solidificação da UNICAMP, utilizando o método da fusão sob gás inerte, detecção por infravermelho para oxigênio e diferença da condutividade térmica para nitrogênio, utilizando-se um analisador de gases LECO TC-400.

A densidade das amostras foi obtida usando-se uma balança analítica Ohaus, modelo Explorer e o kit de determinação de densidade, utilizando o princípio de Arquimedes.

Para preparar a liga para o processo de conformação mecânica, foi realizado um tratamento térmico de homogeneização em alto-vácuo $\left(\sim 10^{-7}\right.$ Torr ou $\left.\sim 10^{-5} \mathrm{~Pa}\right)$ a $1000{ }^{\circ} \mathrm{C}$ durante 24 horas, com taxa de aquecimento e resfriameno de $10{ }^{\circ} \mathrm{C} / \mathrm{min}$. A conformação mecânica se deu por um processo de laminação a quente, que foi realizado a $1000{ }^{\circ} \mathrm{C}$, utilizando-se uma máquina FENN, modelo 5 Mill, pertencente no Laboratório de Fusão por Feixes de Elétrons e Tratamentos Termomecânicos da UNICAMP. O lingote sofreu redução de aproximadamente $20 \%$ de sua espessura em cada passagem, até atingir $2 \mathrm{~mm}$ de espessura. Após a conformação mecânica, um novo tratamento térmico de solubilização foi realizado um em alto-vácuo $\left(\sim 10^{-7}\right.$ Torr ou $\sim 10^{-5} \mathrm{~Pa}$ ) a $1000^{\circ} \mathrm{C}$ durante 24 horas, com taxa de aquecimento e resfriameno de $10{ }^{\circ} \mathrm{C} / \mathrm{min}$.

A caracterização estrutural foi realizada por medidas de difração de raios-X (DRX). Os difratogramas foram obtidos utilizando-se o método do pó, no modo de tempo fixo, com passo de $0,02^{\circ}$ e tempo de permanência de $1,6 \mathrm{~s}$, utilizando radiação $\mathrm{K}_{\alpha}$ do cobre $(\lambda=1.544 \AA$ Á), num difratômetro Rigaku, modelo D/Max 2100/PC.

A caracterização micoestrutural foi realizada por microscopia óptica (MO), utilizando-se um microscópio Olympus, modelo BX51M e por microscopia eletrônica de varredura (MEV), utilizando-se um microscópio Carl Zeiss modelo EVO/LS15. Para obter as micrografias, as amostras foram preparadas de acordo com o processo metalográfico padrão [24]. 


\section{RESULTADOS E DISCUSSÃO}

A Fig. 1 mostra o lingote da liga Ti-10\%pNi, obtido por fusão à arco dos metais precursores, Ti e Ni, e a imagem obtida por EDS dos elementos Ti e Ni distribuidos na liga preparada. Pode-se observar, na parte (a) da Fig. 1, um lingote de coloção cinza brilhante, indicando baixa ou nenhuma contaminação superficial por oxigênio e nitrogênio. É possível observar na parte (b) da Fig.1, uma distribuição homogênea dos elementos de liga, não apresentando formação de precipitados, ou seja, regiões com maiores concentrações de um determinado elemento. Este resultado mostra que a fusão foi completa e homogênea, sem a formação de segregados.

A Tabela 1 apresenta a composição química da amostra da liga Ti-10\%pNi após a fusão e a Tabela 2 mostra os valores obtidos para os elementos intersticiais pesados (oxigênio e nitrogênio) e a densidade, em todas as condições de processamento.

O resultado da análise química mosta que a liga apresenta quantidade de níquel bem próxima ao valor estequiométrico. Segundo a norma ASTM F2063 [25] que traz as especificações padrão com respeito à liga Nitinol utilizada para implantes e dispositivos médicos, o teor máximo de ferro admito é de 0,05\% e a soma de oxigênio e nitrogênio deve ser superior a 0,05\%, valores bem inferiores à liga em questão, podendo trazer interferência em suas propriedades mecânicas e resistência à corrosão. Segundo a análise dos elementos intersticiais oxigênio e nitrogênio observa-se que as amostras apresentam aumento na quantidade destes elementos, principalmente nitrogênio, após a laminação a quente, provavelmente em função deste processo não ser realizado em vácuo e sim em atmosfera controlada de argônio, mantendo-se estável após o tratamento térmico de solubilização. Apesar dos valores elevados, eles estão bem abaixo do limite de solubilidade, em torno de $14,5 \%$ para oxigênio e 6,2\% para nitrogênio []ㅡ. Pode ser observado, a partir dos resultados de densidade, que a adição de níquel aumentou a densidade em relação ao Ti-cp $\left(4,51 \mathrm{~g} / \mathrm{cm}^{3}\right)$. Isto ocorre devido a grande diferença de densidade com o níquel $\left(8,91 \mathrm{~g} / \mathrm{cm}^{3}\right)$ [26]. As condições de processamento não influenciam de maneira significativa os valores de densidade, indicando que os componentes estão próximos do valor estequiométrico (como mostrado pela análise química), certificando a boa qualidade das amostras produzidas.

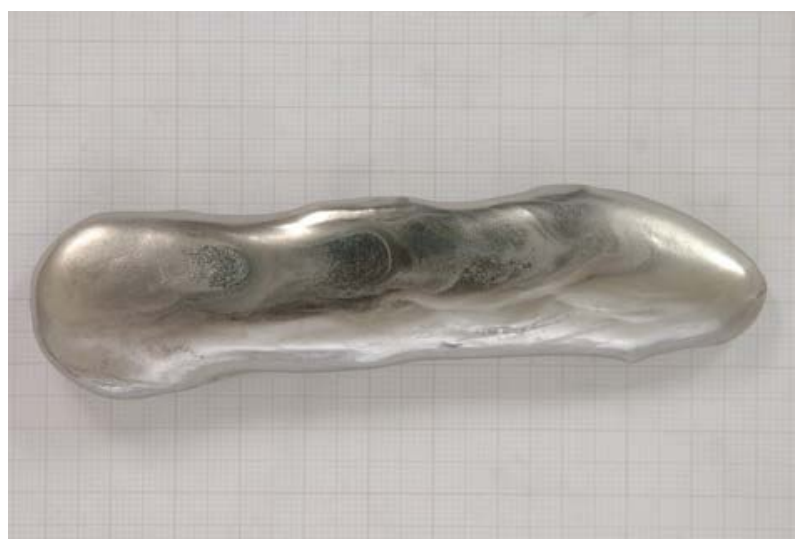

(a)

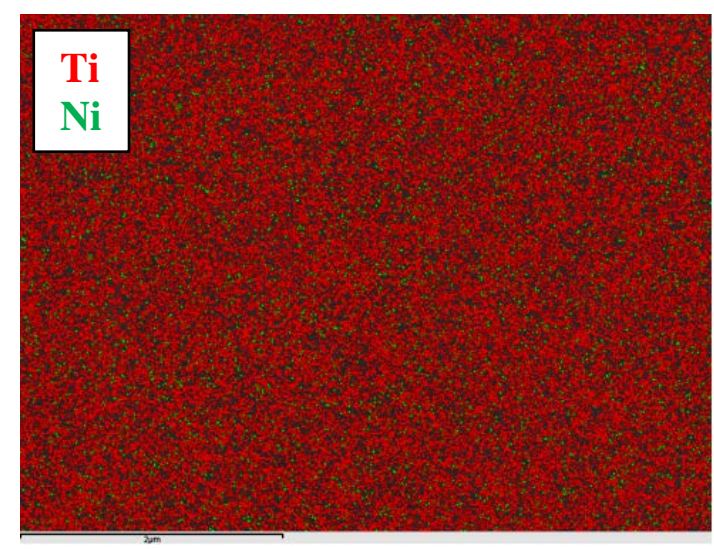

(b)

Figura 1: Fotografia do lingote da liga Ti-10\%pNi (a) e mapeamento, por EDS, mostrando a distribuição dos elementos titânio e níquel na liga Ti-10\%pNi (b).

Tabela 1: Análise química da amostra da liga Ti-10\%pNi, após a fusão.

\begin{tabular}{lllllllll}
\hline Elemento & $\mathrm{Ti}$ & $\mathrm{Ni}$ & $\mathrm{Fe}$ & $\mathrm{Al}$ & $\mathrm{Mn}$ & $\mathrm{C}$ & $\mathrm{O}$ & $\mathrm{N}$ \\
\hline \%p & balanço & 10,03 & 0,1 & 0,018 & 0,034 & 0,38 & 0,36 & 1,54 \\
\hline
\end{tabular}

Os difratogramas para as amostras da liga Ti-10\%pNi nas três condições de processamento estão mostrados na Fig. 2, comparando-se com o difratograma do titânio comercialmente puro. Os difratogramas apresentam picos característicos das fases $\alpha$ do Ti além da fase intermetálica $\mathrm{Ti}_{2} \mathrm{Ni}$. As semelhanças entre os difratogramas com relação às posições e altura dos picos mostram que os processamentos e quantidade de elementos intersticiais não alteraram de maneira significativa as proporções das fases. Analisando o diagrama 
de equilíbrio de fases para o sistema binário Ti-Ni [27], observa-se que a adição de 10\% em peso de Ni dá origem à uma nova fase intermetálica. Segundo Nagarajan e colaboradores [27], em ligas Ti-Ni com aproximadamente esta mesma composição, as fases predominantemente presentes são $\alpha, \beta$ e o intermetálico $\mathrm{Ti}_{2} \mathrm{Ni}$, similares ao presente trabalho, embora o processamento seja diferente, pois as amostras foram analisadas com resfriamento rápido. Contudo, Luo e colaboradores [28] obtiveram uma liga Ti-7Ni por meio de sinterização cujo difratograma é similar à liga em questão.

Tabela 2: Teor de elementos intersticiais e densidade, para a liga Ti-10\%pNi, em todas as condições de processamento.

\begin{tabular}{llll}
\hline Condição & Oxigênio (\%p) & Nitrogênio (\%p) & Densidade (g/cm $\mathbf{3}$ \\
\hline Após fusão & $0,36 \pm 0,04$ & $1,54 \pm 0,25$ & $4,71 \pm 0,05$ \\
Após laminação & $0,8 \pm 0,4$ & $2,8 \pm 1,2$ & $4,75 \pm 0,03$ \\
Após tratamento térmico & $0,8 \pm 0,1$ & $2,5 \pm 0,4$ & $4,73 \pm 0,04$ \\
\hline
\end{tabular}

A Fig. 3 mostra as micrografias obtidas por MO e a Fig. 4 por MEV, para as amostras da liga Ti$10 \%$ pNi, em todas as condições de processamento. Pode ser observada uma morfologia composta por estruturas lamelares ou agulhas, características da fase $\alpha$ do titânio numa matriz eutetóide composta da fase alfa e a fase intermetálica $\mathrm{Ti}_{2} \mathrm{Ni}$. Tais micrografias corroboram com os resultados obtidos por DRX. As estruturas maiores observadas após o tratamento térmico de homogeneização mostram que o resfriamento à taxa de $10^{\circ} \mathrm{C} / \mathrm{min}$ foi suficientemente lento para evitar a formação de fases martensíticas fora do equilíbrio.

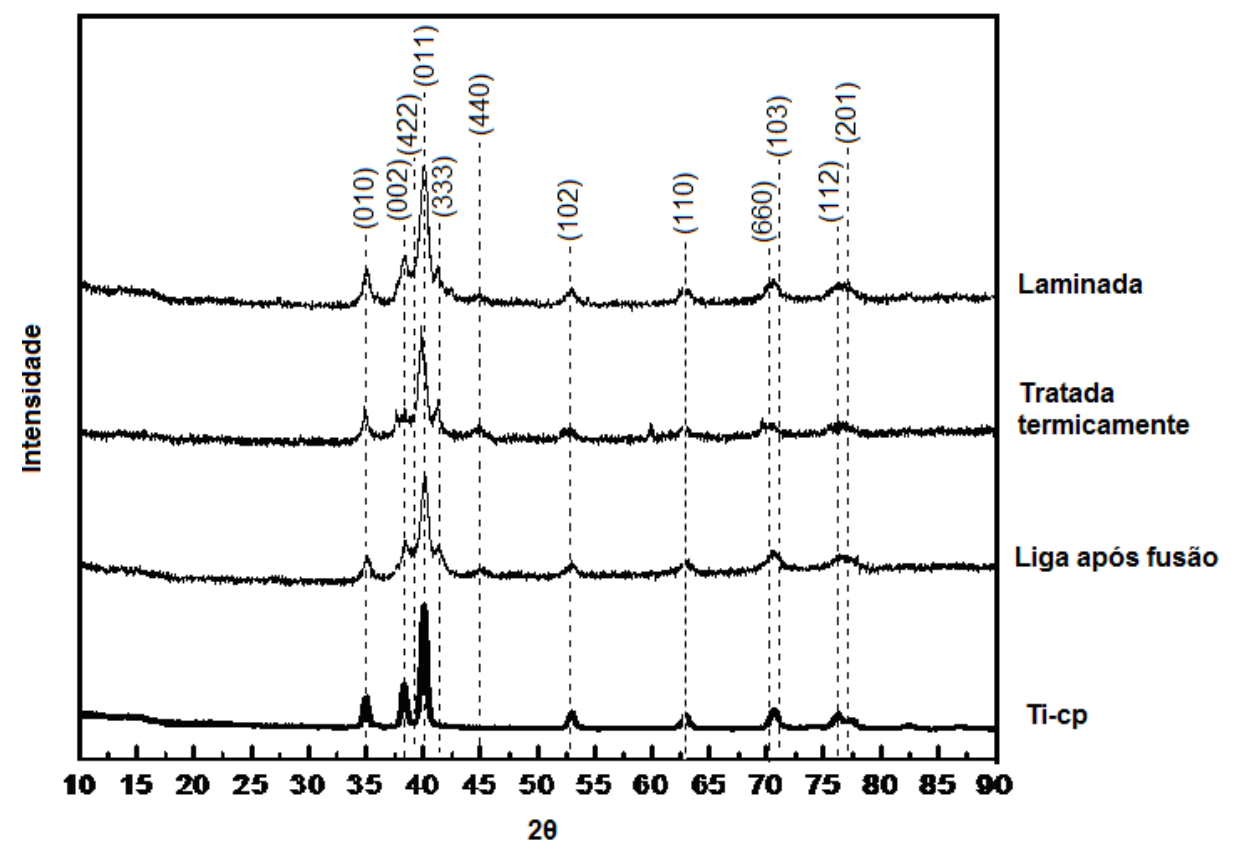

Figura 2: Difratogramas de raios X da liga Ti-10\%pNi após fusão, tratamento térmico e laminação, comparando-se com o Ti-cp, indicando os planos de difração $[\underline{7}, \underline{28}]$.

\section{CONCLUSÕES}

A liga obtida por fusão a arco apresenta a distribuição e composição adequada dos elementos formadores. Os difratogramas de raios-X e as micrografias mostram que em todos os processamentos a liga apresenta predominantemente a fase $\alpha$ do titânio e uma adição de $10 \%$ em peso de níquel provoca a formação de precipitados da fase $\alpha$ numa uma matriz eutetóide composta da fase $\alpha$ e do intermetálico $\mathrm{Ti}_{2} \mathrm{Ni}$. A densidade não é afetada significamente pelo tamanho das microestruturas nem pela quantidade de átomos de oxigênio e nitrogênio em solução sólida presentes nas amostras. São necessários testes de bicompatibilidade e resistência à corrosão para avaliar a possível liberação de níquel e sua citoxicidade. 


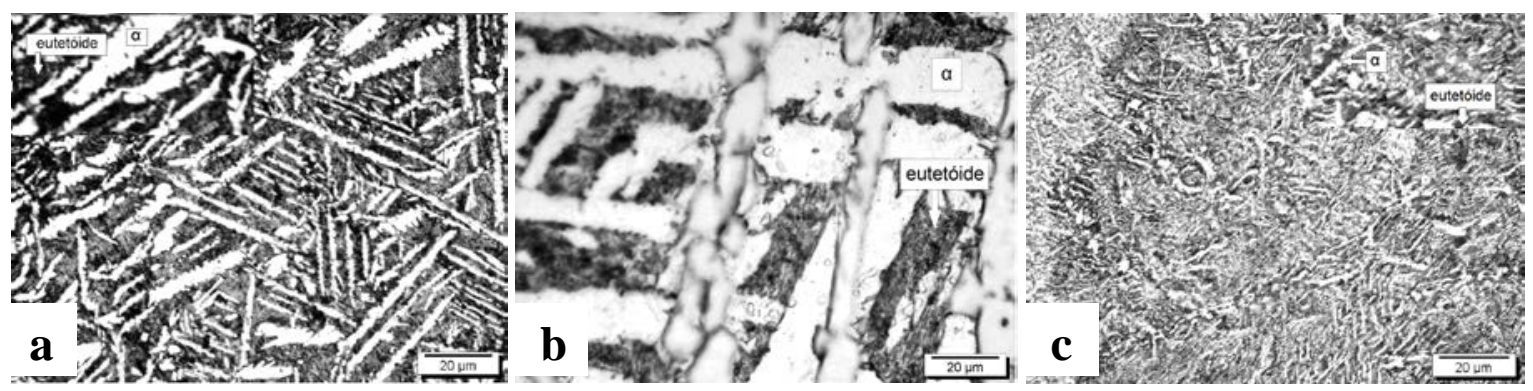

Figura 3: Micrografias ópticas para as amostras da liga Ti-10\%pNi, obtidas após a fusão (a); após tratamento térmico de homogeneização (b) e após laminação a quente (c).
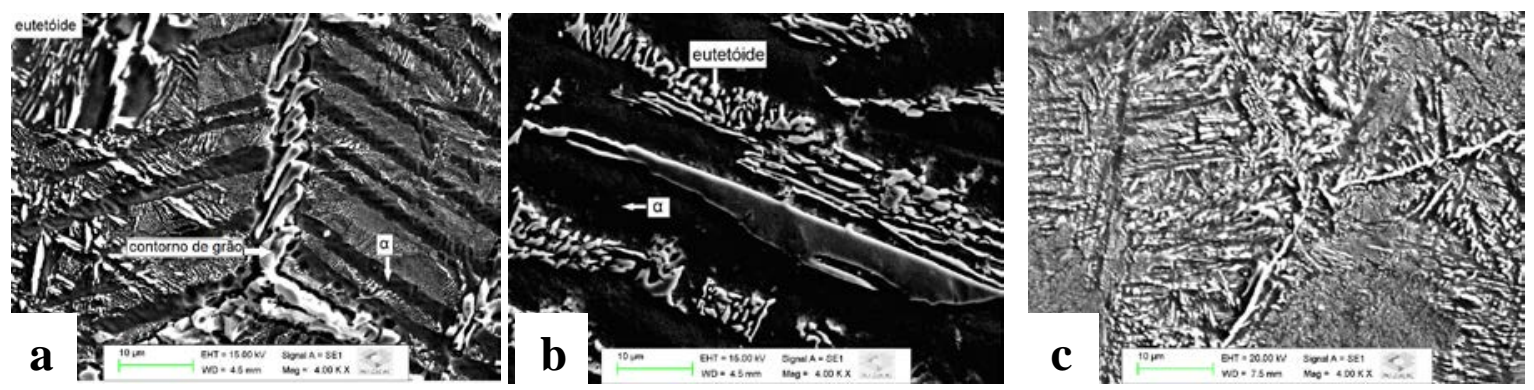

Figura 4: Micrografias obtidas por MEV para as amostras da liga Ti-10\%pNi, obtidas após a fusão (a); após tratamento térmico de homogeneização (b) e após laminação a quente (c).

\section{AGRADECIMENTOS}

Os autores agradecem à CAPES, CNPq e FAPESP, pelo suporte financeiro.

\section{BIBLIOGRAFIA}

[1] LLOYD, A. W. "Biomaterials—where are we going?", Pharmaceutical Science \& Technology Today, v. 1, n.9, pp. 363-364, 1998.

[2] GEETHA, M., et al. "Ti based biomaterials, the ultimate choice for orthopaedic implants - a review", Progress in Materials Science, v. 54, n.3, pp. 397-425, 2009.

[3] BANERJEE, D.,WILLIAMS, J. C. "Perspectives on titanium science and technology", Acta Materialia, v. 61, n.3, pp. 844-879, 2013.

[4] NASAB, M. B., HASSAN, M. R. "Metallic biomaterials of knee and hip" - a review, Trends in Biomaterials and Artificial Organs, v. 24, n.2, pp. 69-82, 2010.

[5] LI, Y., et al. "Cytotoxicity of titanium and titanium alloying elements", Journal of Dental Research, v. 89, n.5, pp. 493-497, 2010.

[6] LEYENS, C., PETERS, M. "Titanium and titanium alloys: fundamentals and applications", New York, Wiley-VCH, 2005.

[7] SILVA, L. M., et al., "Influence of heat treatment and oxygen doping on the mechanical properties and biocompatibility of titanium-niobium binary alloys", Artificial Organs, v. 35, n.5, pp. 516-521, 2011. ISSN 1525-1594.

[8] SILVA, L. M. et al. "Influence of the substitutional solute on the mechanical properties of Ti-Nb binary alloys for biomedical use", Materials Research, v. 15, n.3, pp. 355-358, 2012.

[9] CORREA, D. R. N., et al. "The effect of the solute on the structure, selected mechanical properties, and biocompatibility of Ti-Zr system alloys for dental applications", Materials Science and Engineering: C, v. 34, pp. 354-359, 2014.

[10] VICENTE, F. B., et al. "The influence of small quantities of oxygen in the structure, microstructure, hardness, elasticity modulus and cytocompatibility of Ti-Zr alloys for dental applications", Materials, v. 7, n.1, pp. 542-553, 2014. 
[11] RUIZ, S. L. M., et al. "Preparation and characterization of Ti-8Ta alloys for biomedical applications", Biomedical Materials, 2014.

[12] MARTINS JUNIOR, J. R. S., et al., "Influence of oxygen content and microstructure on the mechanical properties and biocompatibility of Ti-15 wt\%Mo alloy used for biomedical applications", Materials, v. 7, n.1, p. 232-243, 2014.

[13] MARTINS JUNIOR, J. R. S., GRANDINI, C. R. "Structural characterization of Ti-15Mo alloy used as biomaterial by Rietveld method.", Journal of Applied Physics, v. 111, pp. 083535-8, 2012.

[14] MARTINS JUNIOR, J. R. S., et al., "Preparation and characterization of Ti-15Mo alloy used as biomaterial", Materials Research, v. 14, n.1, pp. 107-112, 2011.

[15] CASCADAN, D., BUZALAF, M. A. R., GRANDINI, C. R. "Effect of heat treatment on microstructure and mechanical properties of Ti-5wt-\%Ni alloys for use as biomaterial", International Heat Treatment and Surface Engineering, v. 8, n.3, pp. 107-110, 2014.

[16] BIESIEKIERSKI, A., et al. "A new look at biomedical Ti-based shape memory alloys", Acta Biomaterialia, v. 8, n.5, pp. 1661-1669, 2012.

[17] KHALIL-ALLAFI, J., AMIN-AHMADI, B., ZARE, M. "Biocompatibility and corrosion behavior of the shape memory NiTi alloy in the physiological environments simulated with body fluids for medical applications", Materials Science and Engineering C, v. 30, n.8, pp. 1112-1117, 2010.

[18] WEVER, D. J., et al. "Cytotoxic, allergic and genotoxic activity of a nickel-titanium alloy", Biomaterials, v. 18, n.16, pp. 1115-1120, 1997.

[19] SHABALOVSKAYA,S., ANDEREGG, J., HUMBEECK, J.V. "Critical overview of nitinol surfaces and their modifications for medical applications", Acta Biomaterialia, v. 4, n.3, pp. 447-467, 2008.

[20] KUJALA, S., et al. "Biocompatibility and strength properties of nitinol shape memory alloy suture in rabbit tendon", Biomaterials, v. 25, n.2, pp. 353-358, 2004.

[21] LUO, S. D., et al. "The effect of a small addition of boron on the sintering densification, microstructure and mechanical properties of powder metallurgy Ti-7Ni alloy", Journal of Alloys and Compounds, v. 555, pp. 339-346, 2013.

[22] KRISHNAMURTHY, S., et al. "Beta-eutectoid decomposition in rapidly solidified titanium-nickel alloys", Metallurgical and Materials Transactions A, v. 19,n.1, pp. 22-23, 1988.

[23] CHERN LIN, J. H., LO, S. J., JU, C. P. "Biocorrosion study of titanium-nickel alloys", Journal of Oral Rehabilitation, v. 23, n.2, pp. 129-134, 1996.

[24] KHAMEI, A. A., DEHGHANI, K. "A study on the mechanical behavior and microstructural evolution of Ni-60wt\%-Ti-40wt\% (60Nitinol) intermetallic compound during hot deformation", Materials Chemistry and Physics, v. 123, pp. 269-277, 2010.

[25] ASTM F2063-12, Standard specification for wrought nickel-titanium shape memory alloys for medical devices and surgical implants, ASTM International, West Conshohocken, PA, 2012.

[26] LIDE, D. C. R C. Handbook of chemistry and physics: a ready-reference book of chemical and physical data, 85th, Boca Raton, USA, CRC Press, 2004.

[27] NAGARAJAN, R., AOKI, K., CHATTOPADHYAY, K. "Microstructural development in rapidly solidified Ti-Ni alloys", Materials Science and Engineering: A, v. 179-180, pp. 198-204, 1994.

[28] NAGARAJAN, R., AOKI, K.., CHATTOPADHYAY, K., "Synthesis of nanodispersed phases during rapid solidification and crystallization of glasses in Ti75Ni25 alloys", Metallurgical and Materials Transactions A, v. 28, pp.2223-2231, 1997. 